\title{
A Neuromorphic GAN system for Intelligent Computing on Edge
}

\author{
Chenchen Liu \\ Computer Science and Electrical Engineering, University of Maryland, Baltimore County. \\ ccliu@umbc.edu
}

\begin{abstract}
Recently, artificial intelligence has gained great success in moving toward to edge devices. However, the involved deep neural network (DNN) computations which extremely resource-intensive and power-hungry became a big challenge to the edge computing. The programmable ReRAM based neuromorphic engine opened an opportunity for efficient DNN computing; however, memory utilization and communication latency in the training process implementation is still significant in the current research, and as yet, unmet challenges. The problem becomes more severe in DNN with complex training process such as the generative adversarial network (GAN) which is widely adopted in the intelligence computing on edge. In this work, we target to solve these challenges by designing an efficient GAN computing system on ReRAM neuromorphic engine with a online training framework and an optimized backward computation and a cross-parallel computation flow to execute the training process efficiently. The system performance is evaluated and compared with traditional GPU accelerator, and our results show $2.8 \times$ speedup and $6.1 \times$ energy-saving.
\end{abstract}

\section{ACM Reference Format:}

Chenchen Liu. 2019. A Neuromorphic GAN system for Intelligent Computing on Edge. In Proceedings of The Fourth ACM/IEEE Symposium on Edge Computing (SEC 2019). ACM, New York, NY, USA, 2 pages. https: //doi.org/10.1145/3318216.3363334

\section{INTRODUCTION}

DNN has been proved to be a great success in driving the development of the state-of-the-art artificial intelligence (AI). Recently, GAN emerged and has been widely utilized in the AI application, such as image examples generation, image-to-image translation, video predication, etc. However, the increased computing complexity, esp. the training process makes its computing on edge become a big challenge. Backpropagation is usually utilized in the DNN training process $[1,2]$. It has extremely high requirement in computation resource, and the low speed and high energy cost become big challenges.

Novel computing architecture designs have been extensively explored to achieve high efficient DNN computations, such as FPGA [3], processing-in-memory [4], neuromorphic computing engine [5]. However, these above research still suffers big challenges in efficient online training-they are either incapable to support

Permission to make digital or hard copies of all or part of this work for personal or classroom use is granted without fee provided that copies are not made or distributed for profit or commercial advantage and that copies bear this notice and the full citation on the first page. Copyrights for components of this work owned by others than ACM must be honored. Abstracting with credit is permitted. To copy otherwise, or republish, to post on servers or to redistribute to lists, requires prior specific permission and/or a fee. Request permissions from permissions@acm.org.

SEC 2019, November 7-9, 2019, Arlington, VA, USA

(C) 2019 Association for Computing Machinery.

ACM ISBN 978-1-4503-6733-2/19/11 . \$15.00

https://doi.org/10.1145/3318216.3363334 online training or has inefficient training procedures. In addition, the training process in GAN raises new challenges: it has two learning models are executed simultaneously and requires an adaptive computation process for optimized computing efficiency. Hence, the previous approached cannot be applied directly.

The high density, multiple states and programmable properties of ReRAM (resistive random-access memory) makes it a promising candidate for the implementation of neuromorphic computing systems [6]. In this work, we propose a ReRAM-based neuromorphic GAN system with an online training framework which has multiple models execute simultaneously and an efficient backward computation flow which asynchronously process tasks.

\section{DESIGN METHODOLOGY}

In this section, we built a ReRAM based online training system for GAN which involves multiple learning model executions. The overall architecture design is described in Section 2.1. As is introduced in Section 2.2, an optimized backpropagation processing flow is developed to improve the online training efficiency by decreasing memory utilization and data communication latency.

\subsection{Overall System Design}

Figure 1 shows the overall system design. The adjacent processing units are partitioned into discriminator, generator, and error calculation (i.e., output layer gradient computation) block in GAN implementation. Each processing units are composed by a sea of ReRAM subarrays. There are three operations in the ReRAM arrays: computing, data storage, and look-up-table (LUT). ReRAM arrays are utilized for convolution (and deconvolution) computation and inevitable intermediate data storage in the discriminator and generator blocks. Besides being used in computing and storage, the ReRAM arrays are also employed in LUTs design [7] in the error calculation block. Together with digital circuitry (e.g.,

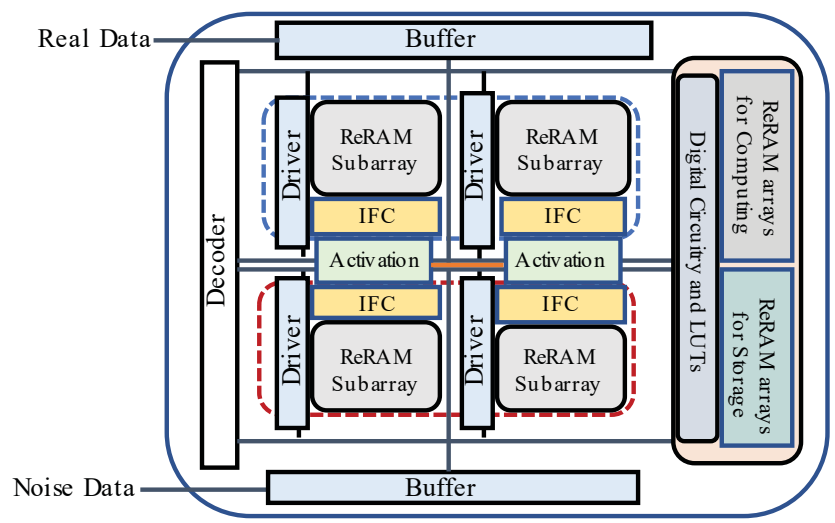

Figure 1: The overall system design of the proposed online training framework. 
adder) [8], they fulfill the complicate cost function. Following the spiking neuromorphic design principle in [5] to reduce design and energy overhead, inputs data are represented by spikes and decoded by the drivers. And integrate-and-fire circuits (IFCs) are employed to transform the sum-of-product current from the tail of ReRAM arrays to readable output pulses [5]. The activation component is utilized for activation function implementation and a ReLU is implemented by subtractors and LUTs in this work [9].

\subsection{Implementation Optimization of Backpropagation Computation}

Backpropagation is utilized as a basic training algorithm and is the major challenge in fulfilling the online training implementation of GAN. It is composed of two processes: forward and backward computations. We develop a parallel forward and an asynchronous backward computation to comprehensively improve the ReRAMbased online training efficiency. By utilizing a four layer neural network as an example, the proposed design is demonstrated in Figure 2.

An Parallel Forward Computation. In the ReRAM based computation, several ReRAM arrays are utilized to implement a convolution (or deconvolution) layer. Generally, higher computing parallelism results in higher speed while heavier hardware design cost. In the backpropagation, the obtained $\Delta W$ (the weight value need to update) are averaged and utilized to update the weights until all the data in a batch are computed. To further accelerate the forward computation, a group of batch data is proposed to execute simultaneously for specified batch size. The optimal solution of the group size will be investigated to achieve the utmost performance in speed and design cost.

An Asynchronous Backward Computation. Previous work investigated ReRAM-based online learning architectures and utilized pipeline structure (i.e., synchronous computing) in training a CNN. Different calculations are executed step by step and the intermediate results are stored in ReRAM modules. This architecture involves considerable overhead in memory utilization. Besides, pipeline bubbles are easily introduced and significant intra-core data communication costs are generated. We target solving these challenges through an asynchronous learning architecture. Starting from the forward process, the input data is given to a neural network

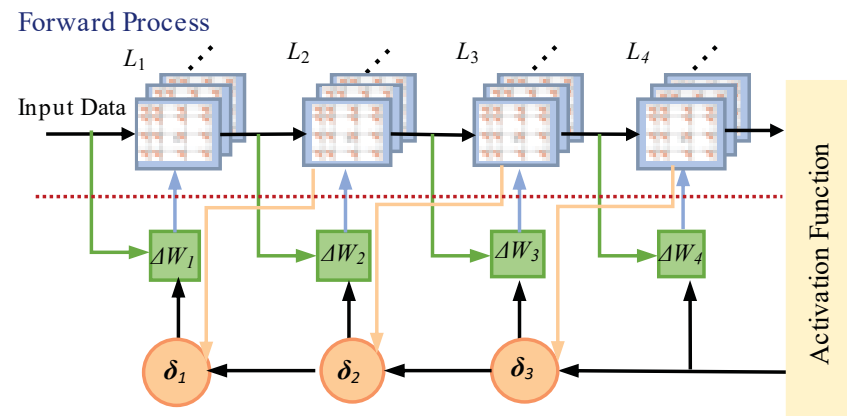

Asynchronous Backward Process

Forward operation $\square \Delta \boldsymbol{W}$ Calculation $\bigcirc$ Error $(\delta)$ Calculation

Figure 2: The proposed backpropagation computing flow. layer (e.g. convolution, fully connected, etc.), which is constructed by several ReRAM arrays to perform the vector-matrix calculation. Instead of introducing memory units to store the output data from each layer, the computed output results are programmed to the ReRAM based computing units for $\Delta W$ calculation immediately. In the backward of gradient calculation, errors from the last layer are fed into the ReRAM-based processing units for the calculation directly without involving in extra memory units and waiting for other unrelated executions (namely, asynchronous execution). The calculated from each layer then enters the ReRAM based processing units for W (new weight value) computation. In this step, results are buffered until all the layers have finished their computations to prevent data distortion and communication traffic congestion in the next layer computation. As such, the training process is accelerated with less design and energy cost.

\section{EVALUATION}

We compared our proposed accelerator with the GPU-based platform. The Nvidia Geforce GTX 1080 GPU is used in this evaluation, and the NVSim simulator is utilized to build the evaluation platform for our proposed design. The experimental results are shown in Table 1 . We can observe that our proposed design can achieve $2.8 \times$ speedup and $6.1 \times$ energy saving compared with the GPU-based platform.

Table 1: Comparison the ReRAM-, GPU-based accelerators

\begin{tabular}{|c|c|c|c|c|}
\hline & \multicolumn{2}{|c|}{ ImageNet } & \multicolumn{2}{c|}{ Energy } \\
\cline { 2 - 5 } & time(h) & speedup & energy(KW/h) & saving \\
\hline This Work & 6.3 & $2.7 \times$ & 0.51 & $6.1 \times$ \\
GPU & 17 & - & 3.1 & - \\
\hline
\end{tabular}

\section{CONCLUSION}

Generative adversarial network (GAN) computing is extremely computationally expensive. To address this issue, we proposed a ReRAM-based neuromorphic accelerator with an optimized backpropagation computing. The proposed accelerator was tested on ImageNet . The proposed accelerator can achieve $2.7 \times$ speedup and $6.1 \times$ energy saving compared with GPU-based computing.

\section{REFERENCES}

[1] Y. LeCun et al., "Gradient-based learning applied to document recognition," in Proceedings of the IEEE, pp. 2278-2324, 2014.

[2] A. Krizhevsky et al., "Imagenet classification with deep convolutional neural networks," in Proceedings of the International Conference on Neural Information Processing Systems (NIPS), pp. 1097-1105, 2012.

[3] W. Zhao et al., "F-cnn: An fpga-based framework for training convolutional neural networks," in IEEE International Conference on Application-specific Systems, Architectures and Processors (ASAP), pp. 107-114, 2016.

[4] P. Chi et al., "Prime: A novel processing-in-memory architecture for neural network computation in reram-based main memory," in ACM/IEEE Annual International Symposium on Computer Architecture (ISCA), pp. 27-39, 2016.

[5] C. Liu et al., "A spiking neuromorphic design with resistive crossbar," in Proceedings of the 52Nd Annual Design Automation Conference, ACM, 2015.

[6] C. Liu et al., "Rescuing memristor-based neuromorphic design with high defects," in Proceedings of the 54th Annual Design Automation Conference (DAC), 2017.

[7] Y. Chen et al., "A novel peripheral circuit for rram-based lut," in 2012 IEEE International Symposium on Circuits and Systems, pp. 1811-1814, May 2012.

[8] C. Liu et al., "A spiking neuromorphic design with resistive crossbar," in ACM/EDAC/IEEE Design Automation Conference (DAC), 2015.

[9] L. Song et al., "Pipelayer: A pipelined reram-based accelerator for deep learning," in IEEE International Symposium on High Performance Computer Architecture (HPCA), 2017. 